\title{
Changes in Health Status Experienced by Women with Gestational Diabetes and Pregnancy-Induced Hypertensive Disorders
}

\author{
CATHERINE KIM, M.D., M.P.H.. ${ }^{1}$ PHYLLIS BRAWARSKY, M.P.H., ${ }^{2}$ \\ REBECCA A. JACKSON, M.D., ${ }^{3}$ ELENA FUENTES-AFFLICK, M.D., M.P.H., ${ }^{4}$ \\ and JENNIFER S. HAAS, M.D., M.S.P.H. ${ }^{2}$
}

\begin{abstract}
Objective: To examine changes in health status among women with gestational diabetes mellitus (GDM) and pregnancy-induced hypertension (PIH).

Methods: We examined perceived health status changes from the prepregnancy, as recalled at 12-20 weeks gestation, to the postpartum period in women with GDM $(n=64)$ vs. unaffected gravidas $(n=1233)$ and women diagnosed with PIH $(n=148)$ vs. unaffected gravidas. We used patient survey and medical record data from a prospective cohort study. Health status measures included the Short Form-36 scales for physical function, vitality, and self-rated health and the Center for Epidemiologic Studies-Depression Scale (CES-D). Multivariate logistic regression models controlled for age, parity, race, education, prepregnancy body mass index (BMI) and exercise levels, food insufficiency, and GDM or PIH during a prior pregnancy.

Results: Women with PIH more often reported a significant decline in vitality (odds ratio [OR] 1.51, 95\% confidence interval [CI] 1.02-2.23) and self-rated health (OR 2.12, 95\% CI 1.193.77) and an increase in depressive symptoms from prepregnancy to postpartum compared with unaffected women (OR 1.84, 95\% CI 1.11-3.05). Decline in self-rated health was partially mediated by cesarean birth and preterm delivery. Similar proportions of women with GDM and unaffected women reported declines in health status measures.

Conclusions: Women with PIH, but not GDM, more often experience significant declines in health status from prepregnancy to postpartum than unaffected women.
\end{abstract}

\section{INTRODUCTION}

G estational Diabetes mellitus (GDM), or glucose intolerance first diagnosed during pregnancy, and pregnancy-induced hypertension (PIH) each affect approximately 5\% of pregnancies in the United States. ${ }^{1,2}$ The effects of these disorders on maternal self-reported health are not

\footnotetext{
${ }^{1}$ Division of General Internal Medicine, Department of Medicine and Division of Women's Health, Department of Obstetrics \& Gynecology, University of Michigan, Ann Arbor, Michigan.

${ }^{2}$ Division of General Medicine and Primary Care, Department of Medicine. Brigham and Women's Hospital and Harvard Medical School, Boston, Massachusetts.

${ }^{3}$ Department of Obstetrics, Gynecology and Reproductive Sciences, and Epidemiology and Biostatistics, and ${ }^{4}$ Department of Pediatrics, University of California, San Francisco, San Francisco, California.

C.K. was supported by an American Diabetes Association Junior Faculty Award. The Women and Infant Starting Healthy Study was supported by the National Institute of Child Health and Human Development (R01 HD37389).
} 
well described, especially for women after they deliver. Such patient-centered measures may offer a broader understanding of maternal health than traditional markers of morbidity and mortality; these measures may distinguish differences between treatment regimens or determine the impact of disease. ${ }^{3}$ This is particularly true for women with GDM and PIH, who are at greater risk than unaffected women for developing future chronic disease ${ }^{4,5}$ but who may have normal glucose and blood pressure levels after delivery. Health status measures may be especially informative for women with GDM. In these women, the benefit of diagnosis has been controversial, with some studies demonstrating lower rates of macrosomia with treatment but increased rates of cesarean birth and little improvement in other measures of morbidity. ${ }^{6}$

To evaluate the influence of GDM and PIH on maternal health status, we examined data from the Women and Infants Starting Healthy (WISH) cohort, a prospective, longitudinal study designed to assess changes in health status over pregnancy. ${ }^{7}$ WISH assessed health status using one of the most widely used measures, the Short Form-36 (SF-36) survey for the Medical Outcomes Study, which surveyed over 22,000 patients in order to develop more practical tools for the routine monitoring of patient outcomes in medical practice. ${ }^{3}$ We hypothesized that women with GDM would more often report declines in health status than women without GDM, and women with PIH would more often report declines in health status than women without PIH. We also hypothesized that any association would be at least partially mediated by cesarean birth or preterm delivery.

\section{MATERIALS AND METHODS}

\section{Population}

Characteristics of WISH have been described previously. ${ }^{7}$ Participants received prenatal care at one of six San Francisco Bay Area hospitals; were $\geq 18$ years of age; spoke English, Spanish, or Cantonese; presented for prenatal care before 16 weeks gestational age; and could be contacted by telephone. Women were enrolled between May 2001 and July 2002. Women who agreed to participate were asked to consent to medical record review and to complete up to four surveys as- sessing health status. The first survey occurred between 12 and 20 weeks gestation and inquired about health status during the month prior to conception (prepregnancy health). The last survey occurred between 8 and 12 weeks postpartum and asked about postpartum health status. Diagnosis of PIH and GDM occurred in each case after the first interview. Postpartum surveys were available for 1480 of the 1657 women (89\%) who delivered at one of the participating sites. For this analysis, we excluded 16 women with both GDM and PIH and 19 women with multiple gestations, for a sample size of 1445 women. The research protocol was reviewed and approved by the institutional review boards of the participating institutions.

\section{Main outcome measures}

We selected the following SF-36 measures representing different dimensions of health and previously demonstrated to change over pregnancy: ${ }^{8}$ (1) the physical function scale (10 items), (2) the vitality scale (4 items), and (3) the self-rated health item (rated as excellent, very good, good, fair, or poor). Scores for the physical function scale and the vitality scale range from 0 to 100 , with a higher score indicating better health status. ${ }^{3}$ We also examined a measure of depressive symptoms, the short-form Center for Epidemiologic Studies-Depression Scale (CES-D) to screen for depressive symptoms (10 items, with a maximum score of 30)..$^{9}$

To ensure that we examined a clinically significant decline in health status, we chose to analyze poor function, defined as a score below the 25th percentile for women of reproductive age based on national normative data. ${ }^{3,9}$ Therefore, we defined a decline in health status from prepregnancy to postpartum as physical function scores $>85$ prepregnancy but $\leq 85$ in the postpartum; vitality scores $>45$ prepregnancy to $\leq 45$ postpartum; self-rated health that was good, very good, or excellent prepregnancy to fair or poor postpartum; and depression scores $\leq 10$ prepregnancy to $>10$ postpartum.

Under PIH, we included gestational hypertension, preeclampsia, and eclampsia. ${ }^{10}$ Under GDM, we included women with glucose intolerance first recognized during pregnancy but not women with previous type 1 or $2 \mathrm{DM} .{ }^{11}$ The working definitions of PIH and GDM may vary between practices. Because we were concerned in 
part with the effect of labeling with these diagnoses and subsequent treatment decisions, we applied the diagnoses of PIH and GDM if they were recorded in the medical record.

\section{Analyses}

The main comparisons were (1) the proportion of women with GDM experiencing a decline in health status from prepregnancy to postpartum vs. the proportion of unaffected women (women without GDM or PIH) with a decline in health status from prepregnancy to postpartum and (2) the proportion of women with $\mathrm{PIH}$ experiencing a decline in health status from prepregnancy to postpartum vs. the proportion of unaffected women (women without GDM or PIH) with a decline in health status from prepregnancy to postpartum. Chi-square tests for categorical variables and log-rank tests for linear variables were used to examine characteristics associated with GDM, $\mathrm{PIH}$, and changes in health status. We then created multivariate logistic regression models for each domain of self-reported health status (physical functioning, vitality, self-rated health, and depressive symptoms). Covariates included in the multivariate models were either significantly associated with GDM or PIH in bivariate associations or included a priori as associated with health status. Multivariate models examining the association between GDM and health status included age, race, education, prepregnancy weight, prepregnancy exercise, parity, prior history of GDM, and insufficient money to pay for housing or food in the past year. Multivariate models examining the association between PIH and health status included age, race, education, prepregnancy weight, prepregnancy exercise, parity, and prior history of PIH. Other candidate covariates included alcohol use, presence of chronic disease, marital status, and cigarette use, but these did not significantly differ between affected and unaffected women and were not included in the final models.

Ten women were noted as having GDM in their medical record but denied having it in the survey, and 56 women were noted as having PIH in their chart but did not report this in the survey. We conducted sensitivity analyses that controlled for report of the diagnosis of GDM or PIH in the chart but not in the patient survey, but these did not change the point estimates, and so these women were included as cases. We also conducted sensitivity analyses that examined the effect of

Table 1. Description of Sample $(N=1445)$ by Gestational Diabetes Mellitus (GDM), Pregnancy-Induced Hypertension (PIH), or Neither Condition

\begin{tabular}{|c|c|c|c|}
\hline & $\begin{array}{c}G D M \\
(\mathrm{n}=64)\end{array}$ & $\begin{array}{c}\text { PIH } \\
(\mathrm{n}=148)\end{array}$ & $\begin{array}{c}\text { Neither } \\
\text { condition } \\
(\mathrm{n}=1233)\end{array}$ \\
\hline Median age in years (range) & $30(18-41)$ & $30(18-40)$ & $30(18-45)$ \\
\hline Median parity (range) & $1(0-6)$ & $0(0-5)$ & $1(0-6)$ \\
\hline \multicolumn{4}{|l|}{ Race, $\%$} \\
\hline Latina & 50.0 & 35.1 & 34.7 \\
\hline White & 25.0 & 30.4 & 34.1 \\
\hline African American & 17.2 & 18.9 & 16.6 \\
\hline Asian/other & 7.8 & 15.5 & 14.6 \\
\hline \multicolumn{4}{|l|}{ Education, \% } \\
\hline Less than high school & 23.4 & 11.5 & 15.5 \\
\hline High school/some college & 46.9 & 52.0 & 40.0 \\
\hline College graduate or more & 29.7 & 36.5 & 44.5 \\
\hline Prepregnancy body mass index (BMI), \% & * & * & \\
\hline Normal/underweight $\left(<25 \mathrm{~kg} / \mathrm{m}^{2}\right)$ & 34.4 & 40.5 & 62.5 \\
\hline Overweight $\left(25.0-29.9 \mathrm{~kg} / \mathrm{m}^{2}\right)$ & 20.3 & 27.7 & 22.6 \\
\hline Obese $\left(\geq 30 \mathrm{~kg} / \mathrm{m}^{2}\right)$ & 45.3 & 31.8 & 14.9 \\
\hline No exercise during month prior to pregnancy, \% & 28.1 & $16.2^{*}$ & 23.6 \\
\hline Insufficient money for food or housing, $\%$ & $25.0^{*}$ & 18.2 & 14.7 \\
\hline History of GDM in prior pregnancy, $\%$ & $21.9^{*}$ & 1.3 & 2.7 \\
\hline History of PIH in prior pregnancy, $\%$ & $17.2^{*}$ & $16.9^{*}$ & 4.9 \\
\hline Cesarean birth, \% & $32.8^{*}$ & $34.5^{*}$ & 21.8 \\
\hline Preterm delivery, $\%$ & 9.4 & $20.3^{*}$ & 5.9 \\
\hline
\end{tabular}

${ }^{*} p$ value $<0.05$ for difference from women without GDM/PIH. 
baseline depressive symptoms on the decline in vitality and self-rated health, but these also did not significantly change the point estimates and were not included in the final models. We conducted sensitivity analyses examining the health status scales as linear variables but found similar trends and present the results as dichotomous variables, as we wanted to present clinically significant changes. We examined whether any declines began from prepregnancy to the third trimester. Finally, we created propensity scores for GDM and PIH and adjusted for these scores in multivariate models, but this adjustment did not significantly change the point estimates and, therefore, were not included in the final models. All analyses were performed using SAS (version 8.0, 2002).

\section{RESULTS}

Characteristics of participants are illustrated in Table 1. Women with GDM had lower educational attainment and were less likely to have a normal prepregnancy body mass index (BMI) and more likely to have had a history of GDM or $\mathrm{PIH}$ in a prior pregnancy compared with women without GDM. Women with GDM were more likely to report insufficiency of money for food or housing. Women with PIH had lower educational attainment were less likely to have a normal prepregnancy BMI, and were more likely to report a history of PIH. Women with GDM and women with PIH were more likely to undergo cesarean section than unaffected women, but only women with PIH were more likely to have preterm delivery than unaffected women.

In unadjusted comparisons, a greater proportion of women with GDM reported poor physical function and self-rated health prior to pregnancy compared with unaffected women (Table 2). A greater proportion of women with PIH reported poor physical function compared with unaffected women. After delivery, a greater proportion of women with GDM reported poor self-rated health compared with unaffected women, and a greater proportion of women with $\mathrm{PIH}$ reported poorer health across all health status measures compared with unaffected women.

In unadjusted analyses, women with GDM and unaffected women had similar declines in all health status measures from prepregnancy to postpartum (Table 3). Women with PIH more often reported declines in vitality and health and

Table 2. Description of Health Status Measures Prior to Pregnancy and Postpartum by Gestational Diabetes Mellitus (GDM), Pregnancy-Induced Hypertension (PIH), or Neither Condition

\begin{tabular}{|c|c|c|c|}
\hline & $\begin{array}{c}\text { GDM } \\
(\mathrm{n}=64)\end{array}$ & $\begin{array}{c}\text { PIH } \\
(\mathrm{n}=148)\end{array}$ & $\begin{array}{l}\text { Neither condition } \\
\quad(\mathrm{n}=1233)\end{array}$ \\
\hline \multicolumn{4}{|l|}{ Health status measures prior to pregnancy } \\
\hline Poor physical function, $\%$ & $20.3^{*}$ & $14.2^{*}$ & 9.2 \\
\hline Poor vitality, $\%$ & 9.4 & 10.8 & 11.1 \\
\hline Poor/fair self-rated health, $\%$ & $21.9^{*}$ & 10.8 & 11.6 \\
\hline Depressive symptoms, $\%$ & 7.8 & 10.1 & 11.6 \\
\hline \multicolumn{4}{|l|}{ Health status measures postpartum } \\
\hline Poor physical function, $\%$ & 26.6 & $31.8^{*}$ & 22.9 \\
\hline Poor vitality, $\%$ & 26.6 & $39.9^{*}$ & 29.8 \\
\hline Poor/fair self-rated health, $\%$ & $18.7^{*}$ & $16.9^{*}$ & 10 \\
\hline Depressive symptoms, \% & 14.1 & $19.6^{*}$ & 13.5 \\
\hline \multicolumn{4}{|c|}{ Change in health status measure from prior to pregnancy to postpartum } \\
\hline Decline in physical function, ${ }^{\mathrm{a}} \%$ & 17.2 & $25.0^{*}$ & 18.3 \\
\hline Decline in vitality, $\mathrm{b} \%$ & 21.9 & $31.1^{*}$ & 22.7 \\
\hline Decline in self-rated health, ${ }^{\mathrm{c}} \%$ & 10.9 & $12.2^{*}$ & 6.7 \\
\hline Increase in depressive symptoms, ${ }^{\mathrm{d}} \%$ & 12.5 & $16.2^{*}$ & 8.3 \\
\hline
\end{tabular}

${ }^{*} p$ value $\leq 0.05$ for difference from women without GDM/PIH.

a Represents a prepregnancy score $>85$ to a postpartum score of $<85$ of 100 on the physical function scale of the Medical Outcome Study Short-Form-36 (SF-36).

${ }^{b}$ Represents a prepregnancy score $>45$ to a postpartum score of $<45$ of 100 on the SF-36 vitality scale.

'Represents a prepregnancy rating of excellent/very good/good to postpartum rating of fair/poor on the SF-36 self-rated health measure.

${ }^{\mathrm{d}}$ Represents a prepregnancy score of $<10$ to a postpartum score of $>10$ out of 30 on the Center for Epidemiologic Studies Scale. 
Table 3. Odds Ratio (OR), 95\% Confidence Intervals (CI) For Decline in Prepregnancy to Postpartum Health Status ${ }^{a}$

\begin{tabular}{|c|c|c|c|c|}
\hline & Unadjusted OR & Adjusted OR & $\begin{array}{l}\text { Adjusted OR } \\
\text { including } \\
\text { cesarean birth }\end{array}$ & $\begin{array}{l}\text { Adjusted OR } \\
\text { including } \\
\text { preterm delivery }\end{array}$ \\
\hline \multicolumn{5}{|l|}{ Gestational diabetes $^{\mathrm{b}}$} \\
\hline Decline in physical functioning & $0.93(0.47-1.80)$ & $0.92(0.45-1.87)$ & $0.83(0.40-1.71)$ & $0.93(0.46-1.88)$ \\
\hline Decline in vitality & $0.95(0.52-1.75)$ & $0.99(0.51-1.90)$ & $0.98(0.51-1.89)$ & $0.98(0.51-1.91)$ \\
\hline Decline in self-rated health & 3.90) & $1.22(0$ & $1.16(0$ & $1.15(0.46-2.86)$ \\
\hline Increase in depressive symptoms & $1.58(0.73-3.41)$ & $1.22(0.54-2.77)$ & $1.22(0.53-2.78)$ & $1.22(0.53-2.78)$ \\
\hline \multicolumn{5}{|l|}{ Prepregnancy-induced hypertension ${ }^{c}$} \\
\hline Decline in physical functioning & $1.49(1.00-2.21)$ & $1.43(0.94-2.18)$ & $1.30(0.84-2.01)$ & $1.51(0.98-2.32)$ \\
\hline Decline in vitality & $1.53(1.06-2.23)$ & $1.51(1.02-2.23)$ & $1.49(1.00-2.20)$ & $1.36(0.91-2.03)$ \\
\hline f-rated health & $1.94(1.13-3.34)$ & $2.12(1.19-3.77)$ & $1.99(1.11-3.57)$ & $1.78(0.98-3.24)$ \\
\hline Increase in depressive symptoms & $2.15(1.33-3.47)$ & $1.84(1.11-3.05)$ & $1.82(1.10-3.03)$ & $1.77(1.05-2.97)$ \\
\hline
\end{tabular}

aRepresents a prepregnancy score $>85$ and postpartum score of $\leq 85$ of 100 on the physical function scale of the SF-36; change from $>45$ to score of $\leq 45$ of 100 on the vitality scale of the SF-36; change from excellent, very good, or good to fair or poor on the SF-36; and a change from $\leq 10$ to $>10$ out of 30 on the CES-D scale.

bAdjusted for age, race, education, prepregnancy weight, prepregnancy exercise level, parity, insufficient money, and prior history of GDM. PIH.

cAdjusted for age, race, education, prepregnancy weight, prepregnancy exercise level, parity, and prior history of

increase in depressive symptoms than unaffected women (Table 3). After adjustment, GDM was not associated with declines in any health status measures, but PIH was still associated with greater declines in vitality and self-rated health and increase in depressive symptoms (Table 3 ).

We further adjusted for cesarean birth (Table 3 ) and preterm delivery (Table 3) to investigate whether health status declines associated with $\mathrm{PIH}$ were independent of mode of delivery and preterm delivery. Relative risks after adjustment for cesarean birth were essentially unchanged for vitality, self-rated health, and depressive symptoms. However, the addition of preterm delivery to the model resulted in greater declines in the risks for vitality and self-rated health and increase in depressive symptoms.

In sensitivity analyses, we examined whether declines in health were evident in the third trimester. We found worsening self-rated health was evident in the third trimester for women with GDM compared with women without GDM, although the effects did not persist into the postpartum period. Specifically, women with GDM had greater unadjusted odds of a decline from prepregnancy to the third trimester in self-rated health (OR 4.03, 95\% CI 2.17-7.48) than unaffected women, and this persisted after adjustment for the factors noted in Table 3 (OR 3.48, 95\% CI 1.756.92). However, GDM was not associated with declines in any other health status measures in the third trimester.

In contrast, from prepregnancy to the third trimester, women with $\mathrm{PIH}$ were more likely to experience declines in vitality (OR 1.57, 95\% CI 1.09-2.26) and self-rated health (OR 2.63, 95\% CI 1.61-4.29) and an increase in depressive symptoms (2.07, 95\% CI 1.40-3.05) than unaffected women. In adjusted analyses, PIH continued to be associated with significantly greater declines in self-rated health (OR 2.94, 95\% CI 1.71-5.01) and an increase in depressive symptoms (OR 1.86, 95\% CI 1.23-2.81) from prepregnancy to the third trimester compared with unaffected women. These changes persisted into the postpartum period.

\section{DISCUSSION}

The influence of GDM and PIH on maternal health after pregnancy has been uncertain. Because of the return of glucose and blood pressure values to normal levels in the majority of women after delivery, GDM and PIH have been conceptualized largely as being limited to pregnancy. It has also been unclear if women with poorer health prior to pregnancy may be predisposed to develop GDM or PIH or if these diagnoses negatively influence maternal health after delivery. 
Finally, the benefit of screening for GDM has been controversial, with subsequent treatment leading to a lower risk of macrosomia but perhaps a higher risk of cesarean delivery. We found that after adjustment for multiple factors preceding diagnosis, PIH was associated with significantly greater declines in vitality and self-rated health and an increase in depressive symptoms compared with unaffected pregnancies. In contrast, GDM was not.

Previous studies examining PIH and maternal health status have suggested that PIH and depressive symptoms are associated, but the influence of PIH on depressive symptoms was not clear. To our knowledge, an association between $\mathrm{PIH}$ and other domains of maternal health status has not been described. Depressive symptoms early in pregnancy ${ }^{12}$ and job stress have been associated with development of preeclampsia later in pregnancy, ${ }^{13,14}$ although health status after the diagnosis of preeclampsia was not examined. Engelhard et al. ${ }^{15}$ found that women with preeclampsia had higher rates of posttraumatic stress disorder (PTSD) than women without preeclampsia, although the study was cross-sectional and unable to adjust for prepregnancy characteristics. We found that PIH was associated with the subsequent development of depressive symptoms postpartum.

In comparison, the association between GDM and maternal health status has been more extensively examined. In an Australian cohort, Rumbold and Crowther ${ }^{16}$ found that women with GDM had negative perceptions about their health after screening for GDM. In another Australian cohort, however, Daniells et al. ${ }^{17}$ found that women with GDM had higher anxiety levels than women without GDM at the beginning of the third trimester, but that the groups were similar antepartum and 6 weeks postpartum. The authors speculated that any worry initially caused by the diagnosis was adequately addressed by treatment plans. Similarly, Langer and Langer ${ }^{18}$ found no difference in women with and without GDM who were at approximately 37-38 weeks gestation, and Spirito et al. ${ }^{19}$ also found no significant difference during pregnancy. We found that before the diagnosis of GDM, women eventually affected by GDM had poorer self-rated health than unaffected women. We also found that this difference in self-rated health did not persist into the postpartum period, as women with and without GDM were essentially similar. Feig et al. ${ }^{20}$ compared women with and without GDM several years after delivery and found that women with GDM had lower self-reported health status. It was not known if the diagnosis of GDM led to poorer health status, or if poorer health status preceded the diagnosis and was a risk factor for GDM. Our results may have differed because we assessed health status postpartum instead of several years after delivery.

$\mathrm{PIH}$ may have a relatively greater impact on maternal health status than GDM for several reasons. PIH is associated with higher rates of complications than GDM, including higher rates of cesarean birth and preterm delivery. ${ }^{21-24}$ After adjustment for cesarean birth and preterm delivery, the associations between PIH and depressive symptoms, self-rated health, and vitality were somewhat reduced. However, the association between PIH and physical functioning was actually strengthened after adjustment for preterm delivery, so greater rates of preterm delivery would not account for all the association.

Although explanations are speculative, it may also be that women with GDM perhaps perceive greater control over their disease than women with PIH. Specifically, diet, exercise, and insulin use may be effective in reducing blood glucose levels and subsequent macrosomia in GDM. ${ }^{25} \mathrm{Al}-$ ternatively, we may have lacked power to detect a difference between women with and without GDM. For example, over 100,000 women would be necessary to detect a significant difference in the observed change of physical functioning between women with and without GDM. In contrast, the pathophysiology for PIH is less clear, and women affected by PIH subsequently have fewer options for prevention and self-management $^{26}$ and may be more fatalistic and negative about their health. The lack of self-management options for PIH may also explain why women with PIH had greater declines in vitality, selfrated health, and depressive symptoms but more similar trends in physical functioning compared with women with unaffected pregnancies. The domains of self-rated health and depressive symptoms reflect perception of health to a greater degree than actual functioning, and it is perception of health that would be affected by lack of control. It may also be that we lacked the power to detect differences in the physical functioning scale between women with PIH and unaffected 
women. However, given the relatively small absolute declines in physical functioning in unadjusted analysis, a sample of approximately 8000 women would be needed to detect a significant difference between these groups, assuming a two-sided alpha at 0.05 and $80 \%$ power. The lack of significant difference could also reflect the generally high health status levels of the childbearing population after delivery. ${ }^{8}$

Our study has several strengths. Using validated measures, we examined changes in health status that have been accepted as clinically significant. We examined data from a populationbased cohort recruited from several medical centers. We were able to identify a pregnant control group. We were also able to obtain measures of maternal health status before the diagnosis of GDM or PIH was made, so that the knowledge of these disorders could not bias reports of prepregnancy health status. We also adjusted for multiple confounders for the relationship between health status and disorders of pregnancy, particularly race and education.

Limitations include the relatively short period of postpartum follow-up. We were also unable to determine if disease severity, such as gestational hypertension vs. eclampsia, or diet-controlled GDM vs. insulin-requiring GDM, was associated with further changes in health status, although we were able to adjust for preterm delivery, a proxy for greater disease severity. Finally, the differences between declines in health status measures for women with GDM and unaffected women were small. It is possible that we were underpowered to detect them, particularly after multivariate adjustment.

\section{CONCLUSIONS}

We conclude that PIH is associated with declines in vitality and self-rated health and an increase in depressive symptoms that persist after delivery compared with unaffected pregnancies but that GDM is not associated with a greater decline than is usually seen with pregnancy. Future studies should examine whether the differences in postpartum health status are mediated by perception of control and lack of self-management options. Investigations should also examine whether differences in postpartum maternal health status are associated with the subsequent development of chronic disease, specifically diabetes or hypertension, and the influence of health status on hospitalizations and costs. These findings can be used to guide the expectations of women and their providers.

\section{REFERENCES}

1. Ferrara A, Kahn H, Quesenberry C, Riley C, Hedderson $\mathrm{M}$. An increase in the incidence of gestational diabetes mellitus: Northern California, 1991-2000. Obstet Gynecol 2004;103:526.

2. Thadhani R, Stampfer M, Hunter D, Manson J, Solomon C, Curhan C. High body mass index and hypercholesterolemia: Risk of hypertensive disorders of pregnancy. Obstet Gynecol 1999;94:543.

3. Ware JJ. SF-36 Health survey: Manual and interpretation guide. Boston, MA: The Health Institute, 1993.

4. Kim C, Newton K, Knopp R. Gestational diabetes and the incidence of type 2 diabetes: A systematic review. Diabetes Care 2002;25:1862.

5. Sibai B, Sarinoglu C, Mercer B. Pregnancy outcome after eclampsia and long-term prognosis. Am J Obstet Gynecol 1992;166:1757.

6. Brody S, Harris R, Lohr K. Summary of the evidence. Screening for gestational diabetes. Agency for Healthcare Research and Quality. Available at www.ahrq.gov/ clinic/3rduspstf/gdm/gdmsum.htm Accessed December 20, 2004.

7. Haas J, Jackson R, Fuentes-Afflick E, et al. Changes in health status of women during and after pregnancy. J Gen Intern Med 2004;20:45.

8. Otchet F, Carey M, Adam L. General health and psychological symptom status in pregnancy and the puerperium: What is normal? Obstet Gynecol 1999;94:935.

9. Andresen E, Mamgren J, Carter W, Patrick D. Screening for depression in well older adults: Evaluation of a short form of the CES-D (Center for Epidemiologic Studies Depression Scale). Am J Prev Med 1994;10:77.

10. ACOG Committee on Obstetric Practice. ACOG practice bulletin. Diagnosis and management of preeclampsia and eclampsia. American College of Obstetricians and Gynecologists. Int J Gynaecol Obstet 2002;77:67.

11. American College of Obstetricians and Gynecologists Committee on Practice Bulletins. Obstetrics. ACOG practice bulletin. Clinical management guidelines for obstetrician-gynecologists. Gestational diabetes. $\mathrm{Ob}-$ stet Gynecol 2001;98:525.

12. Kurki T, Hiilesmaa V, Raitasalo R, Mattila H, Ylikorkala O. Depression and anxiety in early pregnancy and risk for preeclampsia. Obstet Gynecol 2000; 95:487.

13. Klonoff-Cohen H, Cross J, Pieper C. Job stress and preeclampsia. Epidemiology 1996;7:245. 
14. Landsbergis P, Hatch M. Psychosocial work stress and pregnancy-induced hypertension. Epidemiology 1996;7:346.

15. Engelhard I, van Rij M, Boullart I, et al. Posttraumatic stress disorder after preeclampsia: An exploratory study. Gen Hosp Psychiatry 2002;24:260.

16. Rumbold A, Crowther C. Women's experiences of being screened for gestational diabetes mellitus. Aus NZ I Obstet Gynaecol 2002;42:131.

17. Daniells S, Grenyer B, Davis W, Coleman K, Burgess J, Moses R. Gestational diabetes mellitus: Is a diagnosis associated with an increase in maternal anxiety and stress in the short and intermediate term? Diabetes Care 2003;26:385.

18. Langer N, Langer O. Emotional adjustment to diagnosis and treatment of gestational diabetes. Obstet Gynecol 1994;84:329.

19. Spirito A, Williams C, Ruggerio L, Bond A, McGarvey S, Coustan D. Psychological impact of the diagnosis of gestational diabetes. Obstet Gynecol 1989;73: 562.

20. Feig D, Chen E, Naylor C. Self-perceived health status of women three to five years after the diagnosis of gestational diabetes: A survey of cases and matched controls. Am J Obstet Gynecol 1998;178:386.

21. Hedderson M, Ferrara A, Sacks D. Gestational diabetes mellitus and lesser degrees of pregnancy hy- perglycemia: Association with increased risk of spontaneous preterm birth. Obstet Gynecol 2003;102:850.

22. Casey B, Lucas M, McIntire D, Leveno K. Pregnancy outcomes in women with gestational diabetes compared with the general obstetric population. Obstet Gynecol 1997;90:869.

23. MacKay A, Berg C, Atrash H. Pregnancy-related mortality from preeclampsia and eclampsia. Obstet Gynecol 2001;97:533.

24. Samadi A, Mayberry R, Zaidi A, Pleasant J, McGhee NJ, Rice R. Maternal hypertension and associated pregnancy complications among African-American and other women in the United States. Obstet Gynecol 1996;87:557.

25. American Diabetes Association. Gestational diabetes mellitus. Diabetes Care 2004;27(Suppl 1):S88.

26. Report of the National High Blood Pressure Education Program Working Group on High Blood Pressure in Pregnancy. Am J Obstet Gynecol 2000;183:S1.

Address reprint requests to: Catherine Kim, M.D., M.P.H. 300 NIB, Room 7C13 Ann Arbor, MI 48109

E-mail: cathkim@umich.edu 
This article has been cited by:

1. M. R. Torloni, A. P. Betrán, B. L. Horta, M. U. Nakamura, A. N. Atallah, A. F. Moron, O. Valente. 2009. Prepregnancy BMI and the risk of gestational diabetes: a systematic review of the literature with meta-analysis. Obesity Reviews 10:2, 194-203. [CrossRef]

2. Catherine Kim , Laura N. McEwen, Aruna V. Sarma, John D. Piette, William H. Herman . 2008. Stress Urinary Incontinence in Women with a History of Gestational Diabetes MellitusStress Urinary Incontinence in Women with a History of Gestational Diabetes Mellitus. Journal of Women's Health 17:5, 783-792. [Abstract] [PDF] [PDF Plus] 